\title{
Gestörte Exekutivfunktionen bei Aphasie
}

\section{Gesprächsfähigkeit bei Aphasie}

Menschen mit Aphasie fällt es häufig schwer, Gespräche zu führen. Dies zeigt sich bei manchen Betroffenen bereits beim Austausch über das Wetter. Anderen gelingt es nur schwer, sich in eine Diskussion mit mehreren Personen einzubringen. Häufig werden diese Schwierigkeiten als besonders einschränkend und belastend empfunden. Um Gespräche zu verbessern, wird in der herkömmlichen Aphasietherapie vorrangig an sprachlichen Fähigkeiten gearbeitet, wie zum Beispiel an der Grammatik. Die Einschränkungen in Gesprächen bei Aphasie scheinen jedoch mehr als ein sprachliches Problem zu sein, und sie sind nicht in fehlendem Wissen um korrektes Gesprächsverhalten begründet. Studien haben gezeigt, dass für erfolgreiche Gespräche auch nichtsprachliche Exekutivfunktionen notwendig sind, die durch neuropsychologische Diagnostik in ihrer Leistung direkt erfasst werden können.

\section{INFOBOX}

„Exekutivfunktionen“ fasst als Regenschirmbegriff eine Vielzahl von Kontrollfunktionen zusammen. Diese neuropsychologischen Funktionen ermöglichen es, im Alltag flexibel zu reagieren, Lösungswege zu entwickeln und anzuwenden sowie das eigene Verhalten zu kontrollieren.

\section{Exekutivfunktionen und deren Einfluss auf Gespräche}

Exekutivfunktionen werden in Gesprächen dann benötigt, wenn es beispielsweise darum geht, flexibel auf Fragen zu reagieren, passende Argumente zu entwickeln oder das eigene Verhalten zu kontrollieren und ggf. auf die Situation anzupassen.

Dabei kommen insbesondere die folgenden Komponenten der Exekutivfunktionen zum Einsatz: Monitoring, Umstel-

- Tab.1 Exekutivfunktionen und Auswirkungen auf Gespräche.

\begin{tabular}{|l|l|l|}
\hline Exekutivfunktion & Mögliche Schwierigkeiten bei Beeinträchtigung \\
\hline Monitoring & $\begin{array}{l}\text { - Fehler werden nicht erkannt und nicht korrigiert } \\
\text { - fehlender „roter Faden“ }\end{array}$ \\
\hline $\begin{array}{l}\text { Umstellungs- } \\
\text { fähigkeit }\end{array}$ & $\begin{array}{l}\text { - Haften an vorherigen Inhalten, obwohl Themenwechsel stattfand } \\
\text { " } \text { auf Fragen erfolgen inadäquate Antworten } \\
\text { - verzögerte Reaktion auf Themenwechsel oder Fragen }\end{array}$ \\
\hline Interferenzkontrolle & " schnelle Ablenkbarkeit im Gespräch \\
\hline Arbeitsgedächtnis & " $\begin{array}{l}\text { Vergessen von bereits überlegten Gesprächsbeiträgen } \\
\text { („was wollte ich nochmal sagen?“) }\end{array}$ \\
\hline
\end{tabular}

lungsfähigkeit, Interferenzkontrolle und Arbeitsgedächtnis:

Monitoring Das Monitoring ermöglicht die eigene Kontrolle über das Gesprochene, wodurch es gelingt, die eigenen Fehler, wie Versprecher, zu erkennen und zu korrigieren. Darüber hinaus erlaubt das Monitoring, Gesprächsbeiträge auf ihre Relevanz und ihren Bezug zum aktuellen Thema zu überprüfen und ggf. zu korrigieren.

Umstellungsfähigkeit Damit ist die Fähigkeit gemeint, schnell und flexibel auf veränderte Bedingungen zu reagieren, wie es bei einem Themenwechsel oder einer unvorhersehbaren Frage notwendig ist.

Interferenzkontrolle Die Interferenzkontrolle ist wichtig, um sich im Gespräch auf das Wesentliche zu konzentrieren. Dafür muss zwischen relevanten und irrelevanten Informationen unterschieden werden. Letztere müssen anschließend ausgeblendet werden, wie zum Beispiel die Stimmen von Personen vom Nebentisch.

Arbeitsgedächtnis Das Arbeitsgedächtnis ermöglicht die kurzzeitige Speicherung von Informationen. In Gesprächen ist dies dann relevant, wenn der Zuhörer eigene Argumente kurzzeitig im Gedächtnis behalten muss, bis der Gesprächspartner ausgesprochen hat. Andersherum ist das Arbeitsgedächtnis auch relevant für das Sprachverstehen: Es ermöglicht beim Zuhören, den Anfang eines gesprochenen Satzes in Erinnerung zu behalten, während der Satz zu Ende gesprochen wird.
Beeinträchtigungen der Exekutivfunktionen können zu folgenden Schwierigkeiten in Gesprächen führen ( $\mathbf{T a b} \mathbf{1}$ ).

\section{FAZIT}

Bei einer Aphasie ist die Fähigkeit, Gespräche zu führen, oftmals eingeschränkt. Ursache hierfür können neben sprachlichen Beeinträchtigungen (Wortschatz, Grammatik) auch Störungen der Exekutivfunktionen (oder besondere Anforderung an diese) sein. Der genaue Zusammenhang zwischen Sprache und Exekutivfunktionen sowie effektive Behandlungsmethoden gestörter Exekutivfunktionen bei Aphasie sind bislang kaum erforscht, erfreulicherweise nehmen sich doch in jüngster Zeit verschiedene Fachbereiche dieser Themen an. Für Menschen mit Aphasie, ihre Angehörigen und beteiligte Berufsgruppen kann bereits das Wissen um mögliche Einschränkungen durch exekutive Störungen hilfreich sein: Sie können ihre Auswirkungen auf Gespräche eher erkennen, die Anforderungen in Gesprächen entsprechend verringern und bei Bedarf weitere diagnostische Abklärung anregen.

Lena Spitzer, Aachen

Stefanie Abel, Aachen/Manchester

Weiterführende Literatur finden Sie online unter

http://dx.doi.org/10.1055/s-0042-124429 